\title{
EMD Based Curve Evolution Method for Segmentation of Lungs on CT Scan Images
}

\author{
K. K. Thanammal ${ }^{1}$ and J. S. Jaya Sudha ${ }^{2}$ \\ ${ }^{1}$ Associate Professor, Department of MCA, S.T. Hindu College, Nagercoil \\ ${ }^{2}$ HOD/CS Engineering, SCTC of Engineering, Thiruvananthapuram \\ ${ }^{1}$ kkthanammal@gmail.com, 2jayasudhajs@gmail.com
}

\begin{abstract}
Medical imaging is the technique that is used to produce images of the human body or parts for clinical purpose. The CT image provides thorough information of structure of lungs, which could be used for better surgical preparation for treating Lung Cancer features. This work proposes a method for segmentation of lungs from the given CT images. Here a curve evolutional framework for segmentation is used. The flow fields driving the curves are based on the distributions of features in the inner and outer regions bounded by the curves. The segmentation method is automatic and shows good result.
\end{abstract}

Index terms: histogram, lung lobes, EMD, CT images

\section{Introduction}

Computer Tomography (CT) is one of the most efficient medical diagnostic methods and has currently a widespread usage. Multi-slice CT scanning technology has revolutionized screening of the lungs and inspires necessitate for pulmonary image analysis. Segmentation of the lungs and lobes is a prerequisite for such image analysis in chest CT scans. CT scan is more appropriate for showing the detailed information of the parts of human body and it is used for various applications such as detection, classification etc. The analysis of lungs in CT image is used to detect the airway and the vessel present in the lungs [6]. The human lungs are subdivided into distinct pulmonary lobes separated by thin barriers, called fissures. Each lobe contains separate supply branches for both vessels and airways. The segmentation of anatomical parts is important in the detection of pathological abnormalities present in the lungs. Segmentation of the pulmonary lobes is relevant in many clinical applications. Accurate lung segmentation allows for the detection and quantification of abnormalities within the lungs. The lobes are separately supplied by the first subdivisions of the bronchial tree after the main bronchi. The lobes function independently within the lungs. Segmentation of the pulmonary lobes is relevant in clinical practice and particularly challenging for cases with severe diseases or incomplete fissures. Pulmonary fissure is a boundary between the lobes in the lungs. Its segmentation is of clinical interest as it facilitates the assessment of lung disease on a lobar level. A new approach has been made for segmenting the major fissures in both lungs on thin-section computed tomography (CT) [5]. An image transformation called "ridge map" is proposed for enhancing the appearance of fissures on CT. A curve-growing process, modeled by a Bayesian network, is described that is influenced by both the features of the ridge map and prior knowledge of the shape of the fissure. The process is implemented in an adaptive regularization framework that balances these influences and reflects the causal dependencies in the Bayesian network using an entropy measure. This method effectively alleviates the problem of inappropriate weights of 
regularization terms, an effect that can occur with static regularization methods. The method was applied to segment and visualize the lobes of the lungs on chest CT of 10 patients with pulmonary nodules. Only 78 out of 3286 left or right lung regions with fissures $(2.4 \%)$ required manual correction. The average distance between the automatically segmented and the manually delineated ground truth fissures was $1.01 \mathrm{~mm}$, which was similar to the average distance of $1.03 \mathrm{~mm}$ between two sets of manually segmented fissures. The method has a linear-time worst-case complexity and segments the upper lung from the lower lung on a standard computer is less than 5 minutes. The general problem of histogram based curve evolution is considered. The novel flow fields derived to guide the evolution process are based on using the Earth Mover's Distance for measuring the dissimilarity between two histograms.

\subsection{Histogram-Based Methods}

Histogram-based methods [4] are very efficient when compared to other image segmentation methods because they typically require only one pass through the pixels. In this technique, a histogram is computed from all of the pixels in the image, and the peaks and valleys in the histogram are used to locate the clusters in the image. Color or intensity can be used as the measure.

A refinement of this technique is to recursively apply the histogram-seeking method to cluster in the image in order to divide them into smaller clusters. This is repeated with smaller and smaller clusters until no more clusters are formed.

One disadvantage of the histogram-seeking method is that it may be difficult to identify significant peaks and valleys in the image. In this technique of image classification, distance metric and integrated region matching are familiar.

Histogram-based approaches can also be quickly adapted to occur over multiple frames, while maintaining their single pass efficiency. The histogram can be done in multiple fashions when multiple frames are considered. The same approach that is taken with one frame can be applied to multiple, and after the results are merged, peaks and valleys that were previously difficult to identify are more likely to be distinguishable. The histogram can also be applied on a per pixel basis where the information results are used to determine the most frequent color for the pixel location. This approach segments based on active objects and a static environment, resulting in a different type of segmentation is useful in video tracking.

\subsection{Edge Detection}

Edge Detection is a well-developed field on its own within image processing. Region boundaries and edges are closely related. Since there is often a sharp adjustment in intensity at the region boundaries, Edge detection techniques have therefore been used as the base of another segmentation technique.

The edges identified by edge detection are often disconnected. To segment an object from an image, however, one needs closed region boundaries. Edge is nothing but boundary between two images. Edge detection techniques refer to the identifying and locating the sharp discontinuities in the images.

The shape of an object can be either in terms of its boundary or in terms of the region it occupies. The shape representation based on boundary information requires image edge detection in this approach. 


\section{Methodology}

\subsection{Lung Segmentation using EMD}

Image segmentation is a process of portioning an image into non overlapped, consistent regions that are homogeneous with respect to some chacteristics like intensity, color, tone or texture and more [3]. Here a curve evolutional framework for segmentation is used. The flow fields driving the curves are based on the distributions of features in the inner and outer regions bounded by the curves. So the general problem of histogram-based curve evolution is considered by deriving the novel fields to guide the evolution process based on using the Earth Mover's Distance (EMD) [2] for measuring the dissimilarity between the two histograms.

Active contours $[9,1,8,7]$ techniques were originally based on flow fields derived by integrating region boundary image data such as edge strength. The descriptors integrated within a region may also depend on the region. First there is a need to derive the gradient flow fields with respect to the Earth Mover's Distance metric.

\section{The EMD}

Let $\mathrm{P}=\left\{\mathrm{p}_{1}, \ldots, \mathrm{p}_{\mathrm{N}}\right\}$ and $\mathrm{Q}=\left\{\mathrm{q}_{1}, \ldots, \mathrm{q}_{\mathrm{N}}\right\}$ be the discrete probability distribution on $\mathrm{N}$ bins. Let $\left\{\mathrm{P}_{1} \ldots \mathrm{p}_{\mathrm{N}}=1\right\}$ and $\left\{\mathrm{Q}_{1}, \ldots, \mathrm{Q}_{\mathrm{N}}=1\right\}$ be the corresponding cumulative distribution functions(CDF). Then

$\operatorname{EMD}(P, Q)=\sum_{i=1}^{N}\left|P_{i}-Q_{i}\right|$

\section{Initialization}

1. Initialize two cluster centers $C_{1}$ and $C_{2}$ by choosing randomly from $\left\{Q_{1}, \ldots Q_{M}\right\}$

2. For each $\mathrm{Q}_{\mathrm{i}}$ compare $\operatorname{EMD}\left(\mathrm{Q}_{\mathrm{i}}, \mathrm{C}_{1}\right)$ and $\operatorname{EMD}\left(\mathrm{Q}_{\mathrm{i}}, \mathrm{C}_{2}\right)$. Assign $\mathrm{Q}_{\mathrm{i}}$ to closer cluster centre.

3. Update $\mathrm{C}_{1}, \mathrm{C}_{2}$ by summing the counts of all assigned $\mathrm{Q}_{\mathrm{i}} \mathrm{s}$ :

4.

$$
C_{j}^{\text {mew }}=\sum_{\{i|l a b e l(i)=j|\}} Q_{i}
$$

\subsection{Curve Evolution Based on EMD}

\section{Maximal-Discrepancy Functional}

Let $\Omega$ be a closed curve in a image plane.

Let $C D F_{R}\left(Z_{i}\right)=\operatorname{Prob}$ (feature value in region $\mathrm{R} \leq_{\mathrm{z}_{\mathrm{i}}}$ )

Where $\mathrm{R}=\{$ in, out $\}$ separated by $\Omega$

To measure the discrepancy by EMD

$$
\begin{aligned}
\operatorname{EMD}(\Omega)=\operatorname{EMD}\left(P_{\text {in }}, P_{\text {out }}\right) \\
=\sum_{i=1}^{N}\left\|C D F_{\text {in }}\left(z_{i}\right)-C D F_{\text {out }}\left(z_{i}\right)\right\|
\end{aligned}
$$


To represent the function (4) as a sum of (absolute value of) expressions of the form

$\iint_{\Omega_{\text {out }}} k_{z_{i}}^{\text {out }}\left(x, y, \Omega_{\text {out }}\right) d x d y+\iint_{\Omega_{\text {in }}} k_{z_{\mathrm{i}}}^{\text {in }}\left(x, y, \Omega_{\text {in }}\right) d x d y$

Let us define $V\left(z_{i j} \Omega\right)=C D F_{i n}\left(z_{i}\right)-C D F_{\text {out }}\left(z_{i}\right)$ and

$T_{z}(x, y)=\left\{\begin{array}{l}1, I(x, y) \leq z \\ 0, I(x, y)>z\end{array}\right.$

Where $I(x, y)$ is the feature value at $(x, y)$. Then,

$\operatorname{CDF}_{R}\left(z_{i}\right)=\operatorname{Prob}\left(I(x, y) \leq z_{i} \mid(x, y) \epsilon R\right) \mid=\frac{\iint_{R} T_{z_{i}}(x, y) d x d y}{\iint_{R} 1 d x d y}$

Denote the denominator by

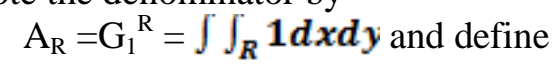

$k_{z_{i}}^{i n}(x, y, \Omega)=g^{i n}\left(x, y, G_{1}^{i n}\right)=\frac{T_{z_{i}}(x, y)}{G_{1}^{i n}}$

Then,

$C D F_{\text {in }}\left(z_{i}\right)=\iint_{\Omega_{\text {in }}} k_{z_{i}}^{i n}(x, y, \Omega) d x d y=\iint_{\Omega_{\text {in }}} \frac{T_{z_{i}}(x, y)}{G_{1}^{i n}} d x d y$.

and in a similar fashion,

$-C D F_{\text {out }}\left(z_{i}\right)=\iint_{\Omega_{\text {out }}} k_{\bar{i}}^{\text {out }}(x, y, \Omega) d x d y=\iint_{\Omega_{\text {out }}}-\frac{T_{z_{i}}(x, y)}{G_{1}^{\text {in }}} d x d y$.

the flow that minimizes

$V\left(z_{i}, \Omega\right)=\iint_{\Omega_{\text {out }}} k_{z_{i}}^{\text {out }}\left(x, y, \Omega_{\text {out }}\right) d x d y+\iint_{\Omega_{\mathrm{fn}}} k_{z_{i}}^{i n}\left(x, y, \Omega_{\text {in }}\right) d x d y$

is given by

$\vec{F}=\left[k_{z_{i}}^{\text {in }}-k_{z_{\tilde{i}}}^{\text {out }}+A_{1}^{\text {in }} H_{1}^{\text {in }}-A_{1}^{\text {out }} H_{1}^{\text {out }}\right] \vec{N}$,

Where $\vec{N}$ is the inward normal to the curve, and $A_{1}^{\text {out }} H_{1}^{\text {out }}$ are as follows:

$$
\begin{aligned}
& A_{1}^{i n}=\iint_{\Omega_{\mathrm{in}}} \frac{\partial g^{i n}}{\partial G_{1}^{i n}}\left(x, y, G_{1}^{i n}\right) d x d y=\iint_{\Omega_{\mathrm{in}}} \frac{-T_{z_{i}}(x, y)}{G_{1}^{i n^{2}}} d x d y \\
& =-\frac{1}{G_{1}^{i n^{2}}} \iint_{\Omega_{\mathrm{in}}} T_{z_{i}}(x, y) d x d y
\end{aligned}
$$

And $H_{1}^{i n}=1\left(G_{1}^{i n}\right.$ integrates 1 over $\left.\Omega_{\text {in }}\right)$. Similarly, $A_{1}^{\text {out }}=\frac{1}{G_{1}^{i i^{2}}} \iint_{\Omega_{\text {out }}} T_{z_{i}}(x, y) d x d x y$. 
Plugging everything in (8), and writing $\mathrm{A}_{\text {in }}, \mathrm{A}_{\text {out }}$ instead of $G_{1}^{\text {in }}, G_{1}^{\text {out }}$, we obtain

$$
\begin{aligned}
& \vec{F}_{z_{i}}(x, y)=\left[\frac{T_{z_{i}(x, y)}}{A_{\text {in }}}+\frac{T_{z_{i}(x, y)}}{A_{\text {out }}}-\frac{C_{\text {in }}}{A_{\text {in }}{ }^{2}}-\frac{C_{\text {out }}}{A_{\text {out }}{ }^{2}}\right] \vec{N} . \\
& C_{\text {in }} \\
& =\iint_{\Omega_{\text {in }}} T_{Z_{i}}(x, y) d x d x y
\end{aligned}
$$

$=$ Number of pixels inside, where $I(x, y) \leq z_{i^{*}}$

$$
C_{\text {out }}=\iint_{\text {out }} T_{Z_{\mathrm{i}}}(x, y) d x d y
$$

$=$ Number of pixels outside, where $I(x, y) \leq z_{i}$

This flow minimizes $N\left(z_{i}, \Omega\right)=C D F_{i n}\left(z_{i}\right)=C D F_{\text {out }}\left(z_{i}\right)$ since we want to maximize

$$
\operatorname{EMD}(\Omega)=\sum_{\mathrm{i}=1}^{\mathrm{N}}\left|\operatorname{CDF}_{\text {in }}\left(\mathrm{z}_{\mathrm{i}}\right)-\operatorname{CDF}_{\text {out }}\left(\mathrm{z}_{\mathrm{i}}\right)\right|_{\text {, }}
$$

The resulting flow is computed as follows:

\section{Algorithm 1.}

1. For every threshold $z_{\mathrm{i}}$, compute

2.

$$
s_{i}=\operatorname{sign}\left|C D F_{\text {in }}\left(z_{i}\right)-C D F_{\text {out }}\left(z_{i}\right)\right|=\left\{\begin{array}{c}
1, C D F_{\text {in }}\left(z_{i}\right) \geq C D F_{\text {out }}\left(z_{i}\right) \\
-1, C D F_{\text {in }}\left(z_{i}\right) \leq C D F_{\text {out }}\left(z_{i}\right)
\end{array}\right.
$$

3. Compute

4.

$$
F_{z_{i}}(x, y)=\left[T_{z_{i}}(x, y)\left(\frac{1}{A_{\text {in }}}+\frac{1}{A_{\text {out }}}\right)-\frac{C_{\text {in }}}{A_{\text {in }}{ }^{2}}-\frac{C_{\text {out }}}{A_{\text {out }}{ }^{2}}\right]
$$

Where $C_{i n}, C_{\text {out }}$ and $A_{\text {in }}, A_{\text {out }}$ are the areas of the inside and outside regions determined by $\Omega$, respectively.

5. The overall flow field is then given by

$$
T(x, y)=\sum_{z_{i}} C\left(-s_{i} F_{z_{i}}(x, y)\right) \vec{N}
$$

Where $\vec{N}$ is the inward normal.

\section{Match to template Functional}

Another useful criterion is to measure how close the distribution inside the curve is to a given template distribution. Let $\mathrm{H}$ be the template histogram which is fixed and independent of the curve $\Omega$. Let $I(\Omega)=\operatorname{EMD}$ (distribution inside $\Omega, \mathrm{H}$ ) be the functional then 
$J(\Omega)=\sum_{\mathrm{i}=1}^{\mathrm{N}}\left|\mathrm{CDF}_{\mathrm{in}}\left(\mathrm{z}_{\mathrm{i}}\right)-\mathrm{CDF}_{\mathrm{H}}\left(\mathrm{z}_{\mathrm{i}}\right)\right|$.

\section{Algorithm 2.}

1. For every threshold $\mathrm{z}_{\mathrm{i}}$ compute $\alpha_{i}=C D F_{H}\left(z_{i}\right)$ and

$s_{i}=\operatorname{sign}\left|C D F_{i n}\left(z_{i}\right)-\alpha_{i}\right|$.

2. Compute$$
F_{Z_{i}}(x, y)=\left[T_{z_{i}}(x, y) \frac{1}{A_{\text {in }}}-\frac{C_{\text {in }}}{A_{\text {in }}^{2}}\right] .
$$

Where $C_{i n}$, is as defined in (11) and $A_{i n^{n}}$ is the area of the inside region determined by $\Omega$.

4. The overall flow field is

$T(x, y)=\sum_{z_{i}}\left(s_{i} F_{z_{i}}(x, y)\right) \vec{N}$,

Where $\vec{N}$ is the inward normal.

\section{Balloon Type Flow}

To choose a more robust alternative by considering a normal flow where the speed is governed by a histogram difference metric. At each point $(\mathrm{x}, \mathrm{y})$ on the curve, a local histogram Hist $(\mathrm{x}, \mathrm{y})$ of feature value is extracted. If $\mathrm{H}$ is the template histogram then flow at $(\mathrm{x}, \mathrm{y})$ is

$$
\vec{F}(x, y)=-e^{-\alpha E M D h\left(\text { Hist }(x, y)_{H} H\right)} \vec{N}
$$

\section{Experimental Results}

This algorithm is applied for any kind of gray level lung images. This algorithm is implemented in MATLAB software. The experiment results in segmenting the right and left lungs. This method finds the lungs by detecting the edges. In the final segmented image it can be specified by detecting its edges. It also uses refinement process to detect the edge.

The original image is shown in Figure 3.1(a). This is given as input .The following list of Figures 3.1(b,c,d,e) show the experimental results obtained by implementing Earth Mover's Distance (EMD) on given CT lung images. Figure 3.1(f) shows the final segmented image. The experimental results show good accuracy.
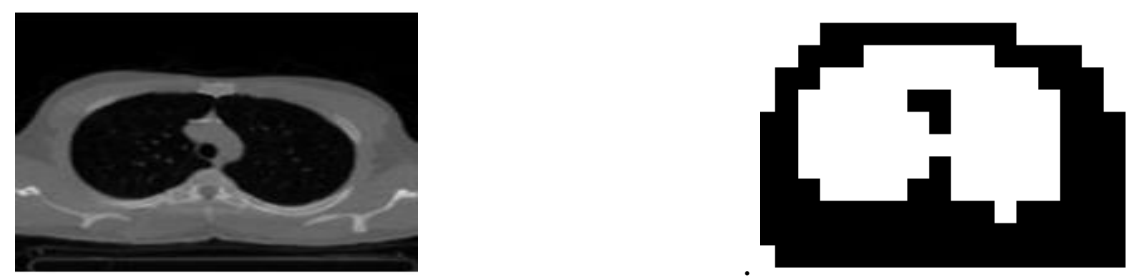

Figure 3.1. (a) Original Input Imag Figure 3.1. (b) Segmented Image Initial Stage 


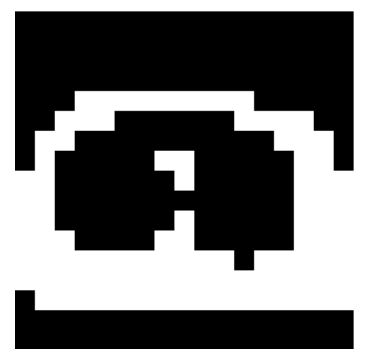

Figure 3.1. (c) Maximum Discrepancy Function

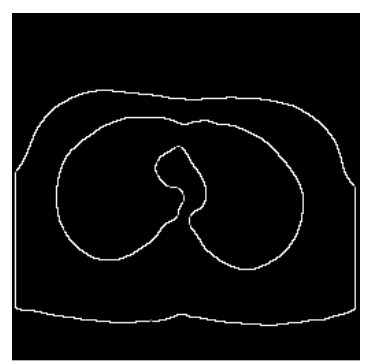

Figure 3.1. (e) Edge Image Refinement Process

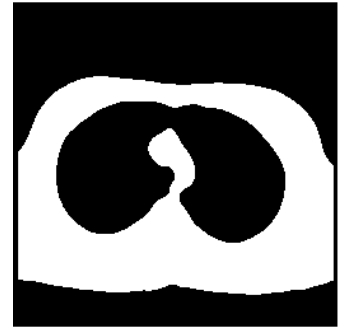

Figure 3.1. (d) Partial Refinement Image

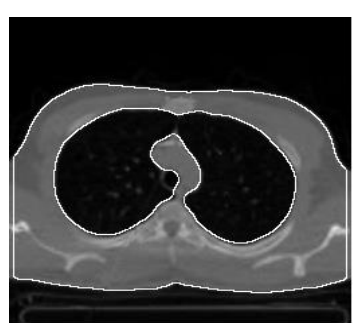

Figure 3.1. (f) Final Segmental Image

\section{Performance Evaluation}

The different CT images are considered as input images as shown in Figure 4.1. The steps for the proposed work are performed for each input image and a final lung segmented image is obtained. This output lung segment image is compared with the accurate lung segment image. Then the accuracy is determined for each input image by comparing the count of pixel values with the accurate lung image. The following Table 4.1 gives the accuracy.

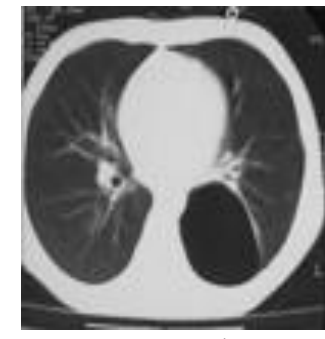

Image 1

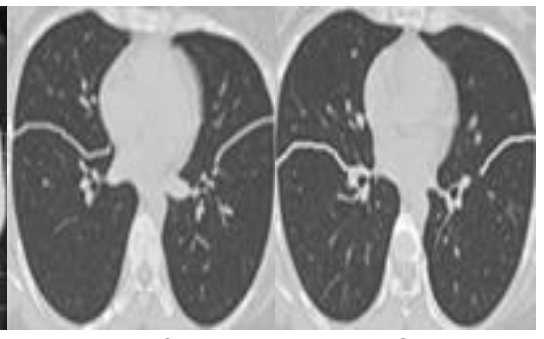

Image 2 Image 3

Figure 4.1. Original Images

Table 4.1. Accuracy of Lobe Segmentation for Various CT Images

\begin{tabular}{|c|c|}
\hline Images & Accuracy \\
\hline 1 & $99.46 \%$ \\
\hline 2 & $99.27 \%$ \\
\hline 3 & $98.07 \%$ \\
\hline
\end{tabular}




\section{Conclusion}

Segmentation of Lung lobes and fissures for surgical planning of treatment of Lung Cancer is proposed in this method. Lung is segmented from chest CT scan images using EMD Based technique. Further processing is carried out to visualize fissure characteristics and each segment inside the lung. Lung fissures are extracted using canny edge detection. The main modules of this work are Preprocessing, Lung Segmentation by EMD Base method and fissure Extraction. Lobe segmentation is carried out by edge tracking and region filling algorithms to differentiate the segments. The segmentation method is automatic and shows good accuracy. The future work concentrates on lobe segmentation to make the accurate result. In this paper, a novel edge detection approach is described. A number of experiments were conducted and the results show that the designed EMD based method.

\section{References}

[1] G. Aubert, M. Barlaud, O. Faugeras, and S. Jehan-Besson, ."Image Segmentation Using Active Contours: Calculus of Variations or Shape , G. Gradients?" SIAM J. Applied Math., vol. 63, no. 6, pp. 2128-2154, 2003.

[2] J. K. Leader, B. Zheng, R.M. Rogers , F.C. Sciurba, A. Perez, B.E.Chapman, S.Patel, C.R. Fuhrman and D.Gur, "Automated Lung Segmentaton in X-ray computed Tomography" Acad. Radiol, vol.10, no.11, pp.1224-1236, 2003.

[3] Nassir Salman "Image Segmentation and Edge Detection Based on Chan- Vese Algorithm" The International Arab Journal of Information Technology, Vol. 3, No. 1. January 2006.

[4] R.C. Gonzalez and R.E. Woods, "Digital Image Processing, $2^{\text {nd }}$ ed. Newyork: Wiley, 2001.

[5] S. A. Kollins, "Computer Tomography of the pulmonary parenchyma and chest wall", Radiolog. Clin.N.Am., vol.15. no.3, pp.297-308, 1977.

[6] Sluimer .I, Schilham A, Prokop M and Van Ginnekan .B, "Computer Analysis of Computed tomography scans of the lung: a Survey", IEEE transactions on Medical Imaging, vol. 25, no. 4, pp. 385-405, April 2006.

[7] T. Chan and L. Vese, "Active Contours without Edges," IEEE Trans. Image Processing, vol. 10, no. 2, pp. 266-277, Feb. 2001.

[8] V. Caselles, R. Kimmel, and G. Sapiro, “Geodesic Active Contours," Int'l J. Computer Vision, vol. 22, no. 1, pp. 61-79, 1997.

[9] Y. Rubner, C. Tomasi, and L. Guibas, "The Earth Mover's Distance as a Metric for Image Retrieval,”Int'l J. Computer Vision, vol. 40, no. 2, pp. 91- 121, 2000.

\section{Authors}

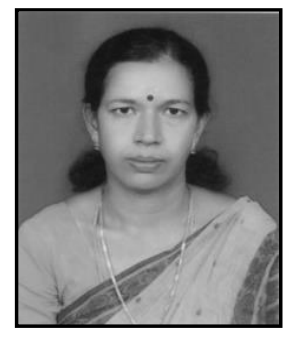

K. K. Thanammal, received the M.Sc (Computer Science) degree from Bharathidasan University, Tiruchirappalli in 1992, M.Phil (Computer Science) degree from Manonmaniam Sundaranar University, Tirunelveli in 1999 and M.Tech (Computer Science) degree from Vinayaka Mission University, Salem in 2007.She is working as an Associate Professor in Computer Science Department at S.T.Hindu College, Nagercoil since 1992. She has 22 years of teaching experience. She has presented and published 6 papers in National and International Conferences and Journals. Her current research interest focuses on Image Processing, Neural Networks, and Medical Images. 


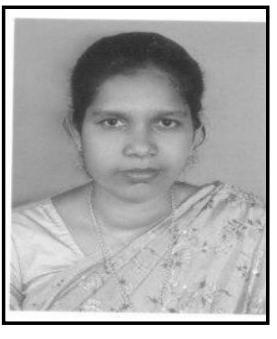

Dr. Jayasudha J. S., received the B.E (Computer Science) degree from R.V.S. College of Engineering, Dindigul in 1994, M.E (Computer Science) degree from National Institute of Technology, Tiruchirappalli in 2002 and Ph.D (Computer Science) degree from Kerala University in 2008. She is working as Asst. Professor and Head in the Department of computer Science \& Engineering, Sree Chitra Thirunal College of Engineering, Thiruvananthapuram, India. She has 16 years of teaching experience. She has presented and published many papers in National and in International Conferences and Journals. Her Current research interest focuses on Image processing, Neural Network, Computer networks. 
International Journal of Bio-Science and Bio-Technology Vol.6, No.5 (2014) 